\title{
EXPLANATION OF ATRIAL BIPHASIC RESPONSES TO ISOXSUPRINE IN THE RABBIT AND GUINEA PIG
}

\author{
MASARU OTSUKA*, MINORU SAKATE**, \\ TAKAO KUBO AND YOSHIMI MISU \\ Department of Pharmacology, Faculty of Pharmaceutical Sciences, Kyoto University, Sakyo-ku, Kyoto \\ Received for publication October 25, 1969
}

Isoxsuprinc, a vasodilator agent, 2-(3 phenoxy-2 propylamino)-1-(p-hydroxyphenyl)1-propanol hydrochloride, was synthesized by Moed and Van Dijk in 1956 (1) and has a $\beta$-stimulatory and adrenolytic action and a papaverine-like or a direct action as reported by Brücke et al. (2) and Ariëns and Simonis (3). Lish et al. (4, 5) attributed a relaxing activity of the drug to the adrenergic $\beta$-stimulatory action in some tissues and the direct action in other tissues when different kinds of non-vascular smooth muscle were used. However, Drinnon and Yclnosky (6) and Manley and Lawson (7) reported that the vasodilator action of isoxsuprine was attributable mainly to the direct action of the drug. Preliminary observations showed atrial biphasic responses-initial stimulatory and then inhibitory responses - to a relatively high dose of isoxsuprine. This study was undertaken to investigate in details a mechanism of atrial biphasic responses to the drug in the rabbit and guinea pig.

\section{METHODS}

About 30 albino rabbits, weighing 2.0 to $2.5 \mathrm{~kg}$, and about 30 guinea pigs, weighing 500 to $600 \mathrm{~g}$, of cither sex, were used. Isolated guinea pig's atria were suspended in 50 $\mathrm{ml}$ of Ringer-Locke's solution at $30^{\circ} \mathrm{C}$ bubbled with $100 \% \mathrm{O}_{2}$ for recording the spontaneous contraction on the smoked drum by means of an isotonic lever. Rabbit's atria were bubbled with $95 \% \mathrm{O}_{2}$ and $5 \% \mathrm{CO}_{2}$ in the same condition. Atrium isolated from about 10 rabbits was fixed on the cork plate in the organ bath for heart muscle (C-509 Takahashi Co.) containing modificd Tyrode's solution at $30^{\circ} \mathrm{C}$ and non-pacemaker potentials were intracellularly recorded in almost the same manncr as previously described (9). High input impedance amplifier (MZ-3A, Nihon Kohden) was connected to universal dual beam oscilloscope ( $\mathrm{VC}_{-1}$, Nihon Kohden). The potential changes were photographed 1hrough a cathode oscilloscope by use of a continuous recording camera (Narishige Scientific Instrument Laboratory). Drugs used were isoxsuprine, dl-noradrenaline hydrochloride, propranolol hydrochloride, atropine sulphate, cocaine and reserpine. Reserpine (3 mg/kg) was injected i.p. in some guinea pigs and the atria were removed 18 to 24 hours later. The concentration of the drugs was expressed in $\mathrm{g} / \mathrm{ml}$.

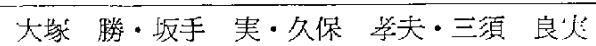

* Present address ; Research Laboratories, Pharmaceutical Division, Sumitomo Chemical Co., LTD., Takarazuka, Hyogo.

** Present address; Department de Farmacologia, Faculdade de Ciẽncias Médicas e Biológicas de Botucalu, Caixa postal 120 Rubião Júnior E.F.S., Estado de São Paulo, Brasil.
} 


\section{RESULTS}

\section{Biphasic effects of isoxsuprine on the isolated atria of the rabbit and guinea pig}

The concentration of isoxsuprine blow $10^{-6}$ did not influence spontaneous atrial contractile frequency and force. Isoxsuprine $10^{-6}$ produced a slight increase in the parameter. As shown in Fig. 1-A and left side of $1-B$, isoxsuprine $5 \times 10^{-5}$ produced increases in atrial contractile frequency and force and these effects reached a maximal point 1 to 3 minutes after the drug application. Maximal increases were 26 and $58 \%(\mathrm{n}=4)$ in the rabbits. Percent increases in the guinea pig were relatively smaller than those in the rabbit. Thereafter, these parameters recovered control levels 4 to 6 minutes after the drug application, and then decreased progressively. As shown in the middlc and right side of Fig. 1-B, 45 minutes after washing out of the drug solution, atrial contractile frequency and force recovered completely and moreover reached and maintained an increased level over control before the drug application. Contractile frecluency of the rabbit atria after washing out of the drug solution was $145.0 \pm 11.8 \%(p<0.05, n=4)$, compared to control $(100 \pm 6.9 \%)$ before the drug application.

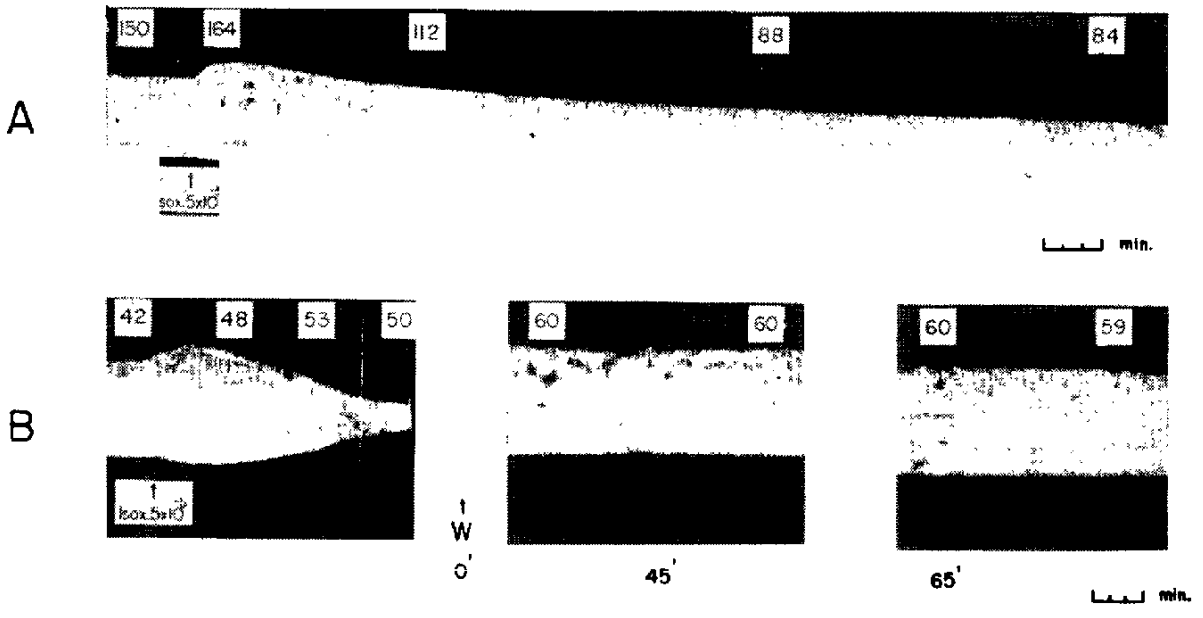

FIC. 1. Biphasic effects of isoxsuprine on the isolated atria of the guinea pig (Trace A) and rabbit (Trace $B$ ).

Numbers at the top of each recording show contractile frequency. Abbreviations: Isox. ; isoxsuprine and $W$; washing out of a test solution. See an increase in contractile frequency and force after washing out of isoxsuprine solution in Trace $B$.

The pretreatment with propranolol $10^{-i}$ blocked completely initial increases in contractile frequency and force caused by isoxsuprine $5 \times 10^{-i}$ and these parameters showed only progressive decreases immediately after isoxsuprine application. Contractile frequency and force after washing out of propranolol-pretreated isoxsuprine solution decreased below control before the drugs application. Contractile frequency decreased to $84 \% \quad(n=2)$ of control in guinea pig's atria.

The pretreatment with cocaine $5 \times 10^{-8}$ did not decrease atrial initial stimulatory response to isoxsuprine $5 \times 10^{-5}$ in the rabbit. Reserpine also did not produce any modi- 
fication in guinea pig's atria. Atropine $10^{-5}$ added 40 minutes after the application of isoxsuprine $5 \times 10^{-5}$ did not influence the later depressive response to the drug in the rabbit.

2. Effects of propranolol, noradrenaline and isoxsuprine after washing out of isoxsuprine solution on the isolated atria of the rabbit and guinea pig

As shown in Fig. 2-A, the administration of propranolol $10^{-7} 30$ minutes after washing out of initially applied isoxsuprine $5 \times 10^{-5}$ solution produced decreases in contractile frequency and force progressively in guinea pig's atria in spite of no changes in these parameters caused by the low concentration of propranolol in standard situation. Percent decreases in contractile frequency and force were 48 and $36 \%(n=3) 10$ minutes after the application of propranolol. As shown in Fig. 2-B, percent increases in contractile frequency and force by the 2 nd administration of noradrenaline $10^{-7} 40$ minutes after washing out of isoxsuprine $5 \times 10^{-5}$ solution were only 30 and $35 \%(\mathrm{n}=2)$ of controls in rabbits. As shown in Fig. 2-C, the 2nd application of isoxsuprine after washing out of the 1st drug solution produced only a very slight initial response in the rabbit, compared to control to the 1st drug application.

A
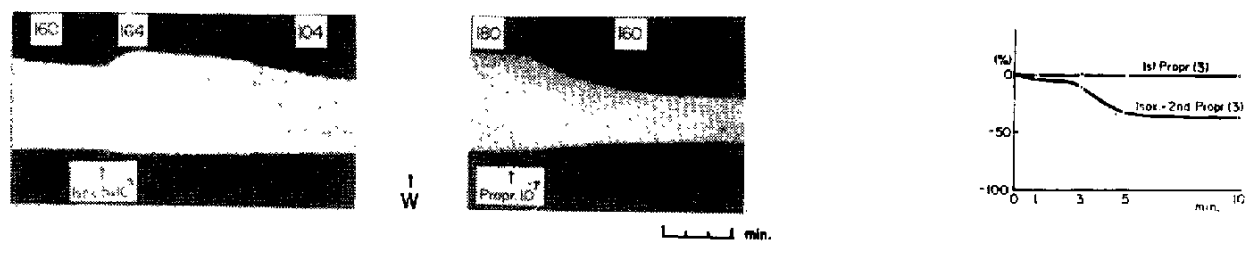

$B$
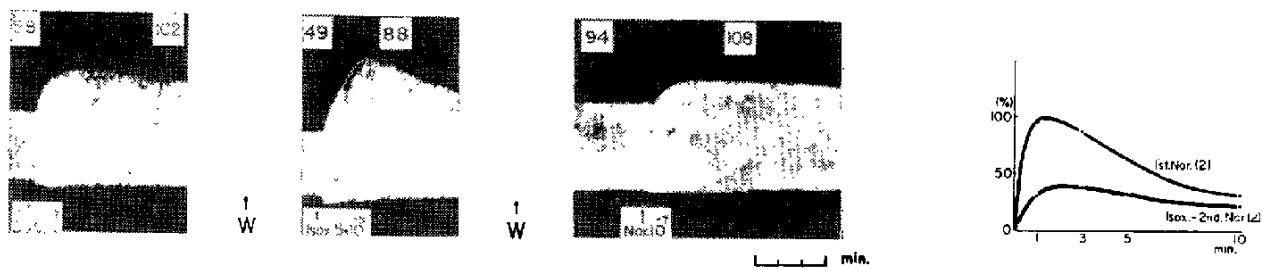

C
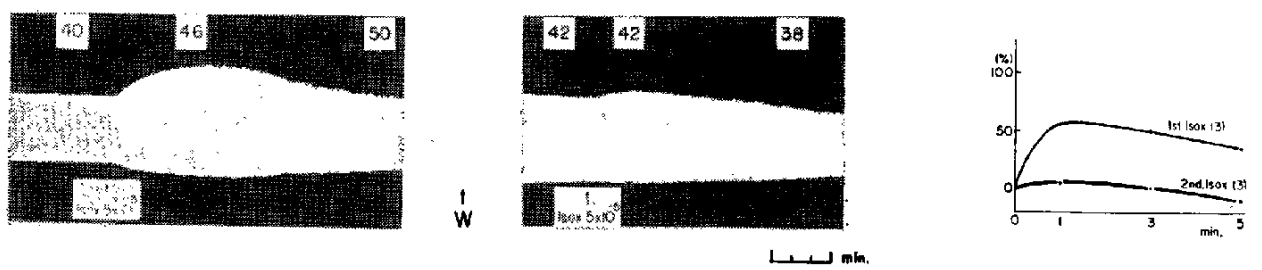

FIG. 2. Effects of propranolol, noradrenaline and isoxsuprine after washing out of isoxsuprine solution on the isolated atria of the guinea pig (Trace A) and rabbit (Traces $B$ and $\mathbf{C}$ ).

Numbers at the top of each recording show contractile frequency. Trace $A$ and $B$ shows a long-lasting $\beta$-stimulatory and $\beta$-blocking action of isoxsuprine, respectively. Trace $\mathrm{C}$ shows a modification of atrial response to the 2 nd application of isoxsuprine. Each figure of the right side shows an average atrial contractile response in each Trace, respectively (Abscissa : time after the drug application, and Ordinate : percent changes in contraction force). Numbers of experiments are shown in parenthesis. Abbreviations : Isox. ; isoxsuprine, Fropr.; propranolol, Nor.; noradrenaline and $W$; washing out of test solutions. 
3. Effect of extracellular high $\mathrm{Na}^{+}$and low $\mathrm{K}^{+}$on a standstill time of contractile frequency in the guinea pig's atria by isoxsuprine

Effect of extracellular high $\mathrm{Na}^{+}$and low $\mathrm{K}^{+}$on the later depressive action of isoxsuprine was investigated in guinea pig's atria in the presence of propranolol $10^{-7}$ as an index of a standstill time of contractile frequency caused by the drug application. $\Lambda$ s shown in Fig. $3-\mathrm{A}$, isoxsuprine $5 \times 10^{-5}$ produced a standstill of atrial contractile frequency about 5 minutes after the drug application in the standard solution. In the equilibrium condition of extracellular $1 / 4 \times \mathrm{K}^{+}$conccntration, atrial contractile frequency decreased and force in-

A

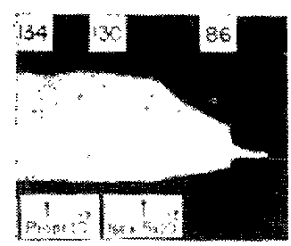

Control

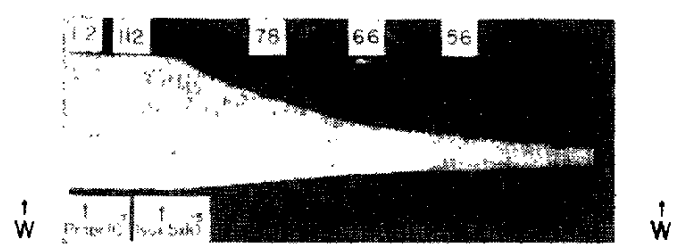

$\mathrm{KC} 1 \mathrm{I} / 4 \mathrm{x}$
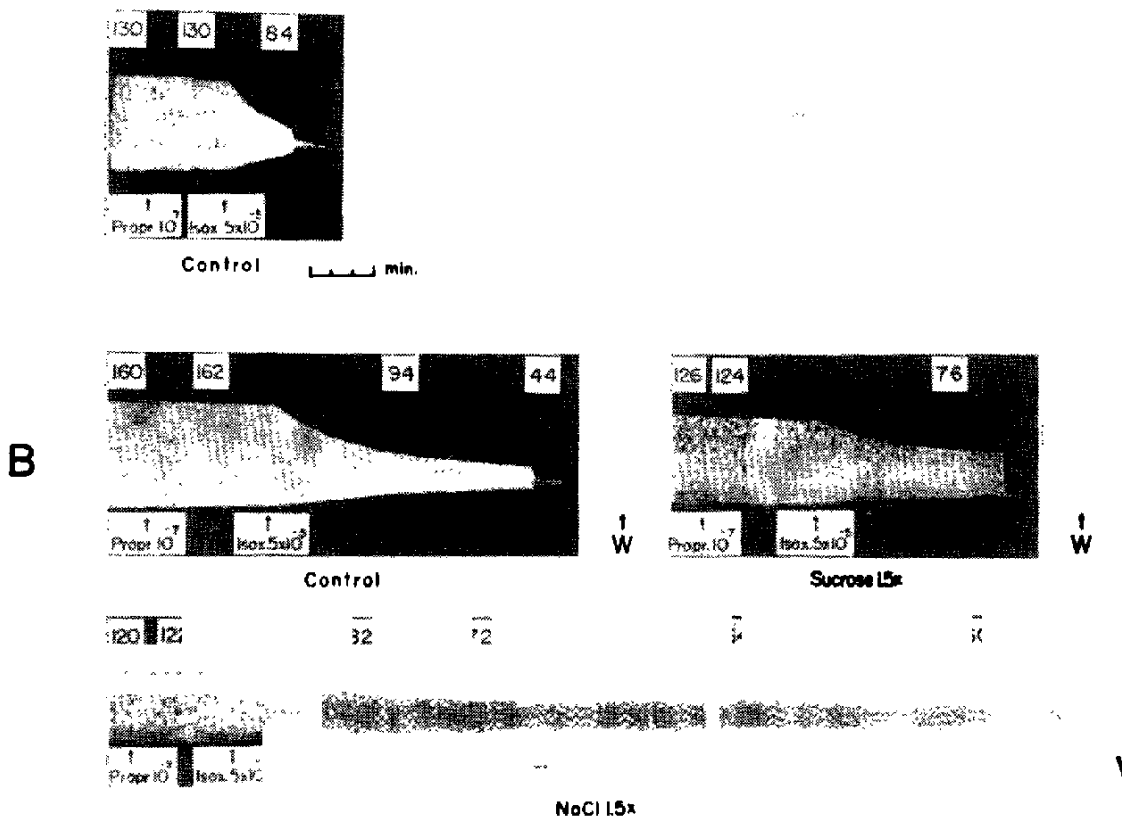

NoCl $1.5 x$

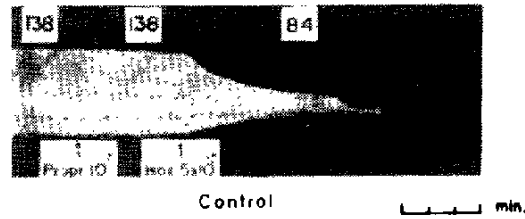

Fig. 3. Typical effect of extracellular low $\mathbf{K}^{+}$(Trace $\mathrm{A}$ ) and high $\mathrm{Na}^{+}$(TraceB) on a depressive action of isoxsuprine in the isolated guinea pig's atria.

Numbers at the top of each recording show contractile frequency. Atria were pretrcated with propranolol $\left(10^{-i}\right)$. Abbreviations : Isox. ; isoxsuprine, Propr. ; propranolol, $\mathrm{W}$; washing out of test solutions, and Sucrose $1.5 \mathrm{x}$; isoosmotic amount of sucrose to extracellular $1.5 \times \mathrm{Na}^{+}$concentration. 

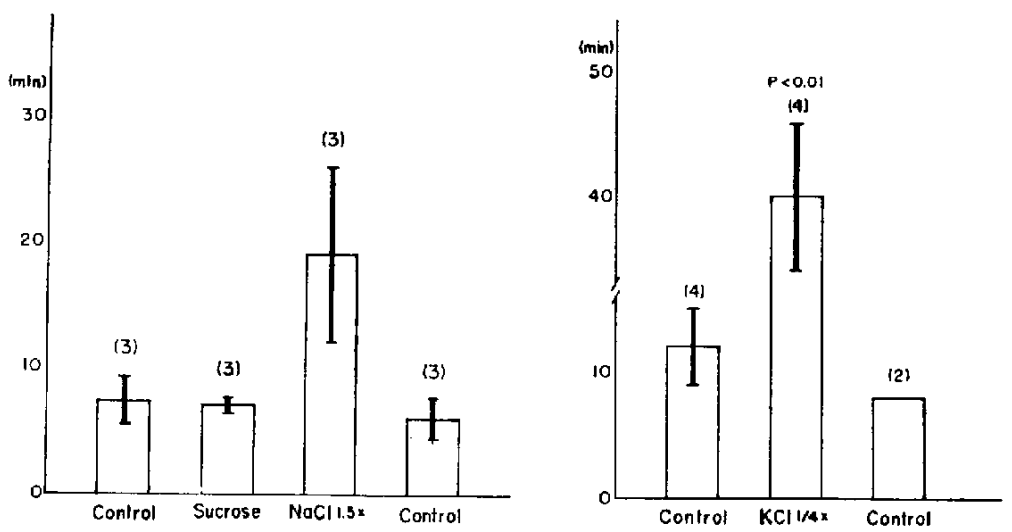

FIG. 4. Effects of extracellular high $\mathrm{Na}^{+}$(left side) and low $\mathrm{K}^{+}$(Right side) on a standstill time of contractile frequency of the guinea pig's ratria after the application of isoxsuprine.

Atria were pretreated with propranolol $\left(10^{-7}\right)$. Ordinate shows the standstill time of contractile frequency caused by isoxsuprine. Numbers of experiments are shown in parenthesis. Vertical bars show standard error. Sucrose means isoosmotic amount of sucrose to extracellular $1.5 \times \mathrm{Na}^{+}$concentration. Experiments were performed in the same atria in the order of left to right columns in each figure.

creased slightly. Standstill time caused by the drug was significantly prolonged (see summarized results in Fig. 4). Again that in the standard solution was almost the same to control.

As shown in Fig. 3-B, in sucrose solution of isoosmotic amount to external $1.5 \times \mathrm{Na}^{+}$ concentration and in the external $1.5 \times \mathrm{Na}^{+}$concentration, atrial contractile frequency and force before the drug application decreased moderately, which may be partially derived from high osmotic pressure. Atrial standstill time by isoxsuprine was prolonged clearly in the condition of external high $\mathrm{Na}^{+}$despite of no prolongation in sucrose solution. Again, in the standard solution in the same atrium, atrial standstill time by the drug was the same to control before changing external solution (also see Fig. 4).

\section{Interaction between external high $\mathrm{Na}^{+}$and effect of isoxsuprine on the non-pacemaker potentials}

in the isolated sponlaneously beating atria of the rabbits

In the presence of propranolol $10^{-7}$, the dose of which was ascertained not to produce any modification on non-pacemaker potentials, isoxsuprine $5 \times 10^{-5}$ produced a gradual decrease in the resting potential and a rounding-off of the upstroke and the peak of the action potential within 10 to 15 minutes after the drug application, as shown in a typical case of Fig. 5-A to -D. The rate of rise and amplitude of the action potentials decreased gradually and the terminal phase of the repolarization proionged markedly. These changes were progressive and the action potentials disappeared completely 30 to 50 minutes after the drug application. Abolished action potentials were started again by washing out of nutrient solution several times. but complete recovery of non-pacemaker potentials was not observed. In a case shown in Fig. 5, an intracellular microelectrode was dislodged after disappearance of the action potentials between D and E. Microelectrode was in- 


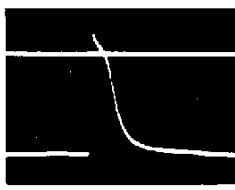

$A$

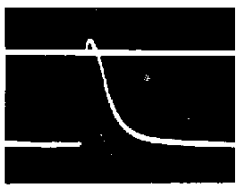

B

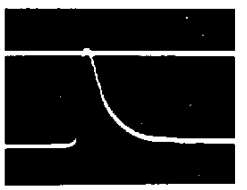

C

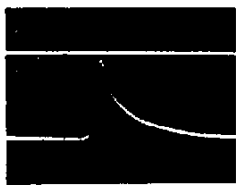

D

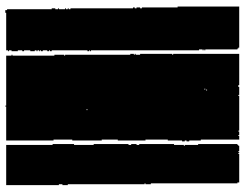

E

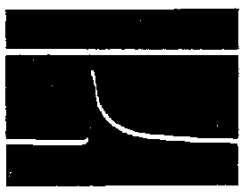

F

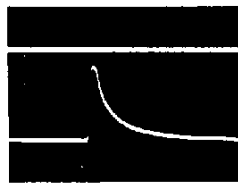

G

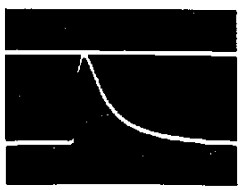

$\mathrm{H}$

FIG. 5. Effect of isoxsuprine on the non-pacemaker potentials in isolated spontaneously beating rabitt's atrium.

Atrium was pretreated with propranolol $\left(10^{-7}\right)$. A : Before. B : 24 minutes after the application of isoxsuprine $5 \times 10^{-5}$. G: 33 minutes after. D : 47 minutes after. E : 51 minutes after. $F: 2$ minutes after the addition of $\mathrm{NaCl}$ $138.5 \mathrm{~mm}$. G : 5 minutes after. H: 10 minutes after. Calibrations : $100 \mathrm{mV}$ and $100 \mathrm{msec}$. Intracellular microelectrode was dislodged between $\mathbf{D}$ and $\mathbf{E}$.

serted again in other fibcr, also in which spontancous firings of the action potential were not observed (Fig. 5-E). Addition of external $1 / 2$ to $1 \times \mathrm{NaCl}$ (totally 217.5 to $277.0 \mathrm{mM}$ ) could restart the action potentials without increasing the resting potential in 3 out of 5 cases. Spontaneous firing of action potentials restarted by high $\mathrm{Na}^{+}$was temporal in 2 out of 3 cases. However, in a case shown in Fig. 5, continuous recovery of the action potential was observed (F-H). In 2 out of 4 cases, non-pacemaker potentials changed but not yet arrested by isoxsuprine could be recovered slightly by the addition of high $\mathrm{Na}^{+}$.

\section{DISCUSSION}

The result of an initial slight stimulatory effect of isoxsuprine on contractile frequency and force in the present expcriment was in accordance with previous observations presented by Brücke et al. (2), and Nash et al. (8) and its adrenergic $\beta$-stimulatory property was also ascertained in the present rcsult as suggested by Ariëns and Simonis (3) and reported by Drinnon and Yelnosky $(6)$. The adrenergic $\beta$-stimulatory action of isoxsuprine was long-lasting in isolated atria. Thirty to 50 minutes after washing out of isoxsuprine 
solution, atrial contractile frequency and force increased clearly compared to the initial level before the drug application and in this situation the application of the low concentration of propranolol produced a progressive decrease in both parameters. On the other hand, washing out of isoxsuprine solution pretreated with propranolol resulted in decreases in both parameters compared to the initial level before the drug application.

Adrenergic $\beta$-blocking activity of isoxsuprine suggested by Ariëns and Simonis (3) and demonstrated by Lish et al. (4) was also confirmed in the isolated atria in the present experiment. Even in the absence of isoxsuprine 30 to 50 minutes after washing out of the drug solution, the 2nd application of noradrenaline produced clearly lesser positive inoand chrono-tropic effects than those of the 1st application of noradrenaline. Lish et al. (4) demonstrated a relatively long duration of relaxing action of isoxsuprine in every type of smooth muscles tested in vivo. This was confirmed in isolated atria in adrenergic $\beta$ blocking action and also in a direct depressive action of the drug. It seems likely that a direct depressive action of isoxsuprine is masked by the long-lasting $\beta$-stimulatory action even after washing out of the drug solution, because depressive effects of isoxsuprine on the non-pacemaker potentials in the presence of propranolol could not be also easily recovered by repeated washing out of the nutrient solution.

$\Lambda$ relatively high dose of isoxsuprinc produced a progressive decrease in atrial contractile frequency and force in the presence of propranolol. Raising external $\mathrm{Na}^{+}$concentration prolonged clcarly a standstill time of atrial contractile frequency caused by isoxsuprine, while the addition of isoosmotic amount of sucrose did not prolong it and addition of $\mathrm{NaCl}$ could recover partially non-pacemaker potentials changed or arrested by isoxsuprine. Also judging from changes in the shape of the transmembrane potentials caused by isoxsuprine, the drug seems surely to inactivate sodium carrying system. However, these effects could not be specific only to isoxsuprine. For example, Cox and West (11) have reported a sodium lactate reversal of quinidine effect on rabbit's atria and Kubo and Misu (10) have reported a partial recovering effect of soidum chloride on non-pacemaker potentials changed by a high dose of propranolol. Lowering external $\mathrm{K}^{+}$also prolonged significantly the standstill time of atrial contractile frequency caused by isoxsuprine. Judging also from changes of non-pacemaker potentials produced by isoxsuprinc, the drug depresses the permeability of the membrane to potassium. Again, effect of low external $\mathrm{K}^{+}$concentration is not specific only to isoxsuprine. For cxample, Holland and Klein (12) have reported the depression of $\mathrm{K}^{42}$ efflux in the presence of quinidine and these observations are not uncommon with many stabilizing drugs (13). Trautwein (14) has pointcd that chemically quite unrelated agents such as quinidine, procaine, diphenhydramine and papaverine have basic common properties in the single fiber experiments. In the present experiment, it was shown that isoxsuprine had a slight $\beta$-stimulatory and $\beta$-blocking action and that a mechanism of the direct depressive action of isoxsuprine was at least due to decreased excitability of atrial muscles. However, it is not clear whether the mechanism of direct action of the drug on vascular smooth muscles is the same to that in heart muscles or not. 


\section{SUMMARY}

Biphasic responses to a relatively high dose of isoxsuprine were observed in the isolated atria of the rabbit and guinea pig.

1. Propranolol blocked initial slight increases in contractile frequency and force by isoxsuprine and revealed only a dircct depressive action of the drug. Adrenergic $\beta$-stimulatory action of the drug was long-lasting. Compared to initial level before the drug application, atrial contractile frequency and force increased significantly after washing out of isoxsuprine solution. Low dose of propranolol applied in this phase produced a progressive decrease in contractile frequency and force.

2. Isoxsuprine produced a slight long-lasting adrencrgic $\beta$-blocking activity. Noradrenaline applied after washing out of isoxsuprine solution produced clearly lesser inoand chrono-tropic actions than controls. The 2 nd application of isoxsuprine in this phase also showed lesser adrenergic $\beta$-stimulatory actions than controls.

3. A standstill time of atrial contractile frequency by isoxsuprine in the presence of propranolol was prolonged in the condition of high external $\mathrm{Na}^{+}$in spite of no prolongation in isoosmotic amount of sucrose solution. The standstill time by the drug was also significantly prolonged in external low $\mathrm{K}^{+}$solution.

4. Isoxsuprine produced decreases in the resting potential, the rate of rise and overshoot of the non-pacemakcr action potential and a prolongation of the terminal phase of the repolarization and finally arrested non-pacemaker potentials completely. Addition of NaCl recovered partially these parameters.

5. It was discussed that isoxsuprine has a slight $\beta$-stimulatory, $\beta$-blocking and a direct depressive action on the isolated atria.

\section{REFERENCES}

1) Moed, H.D. And Van Dijk, J.: Rec. tran. chim. 75, 1215 (1956)

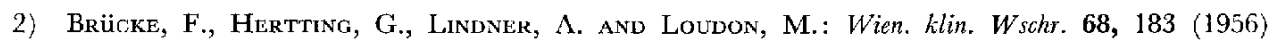

3) Ariëns, E.J. And Simonis, A.M.: Archs int. P.zarmacodyn. Thér. 127, 479 (1960)

4) Lish, P.M., Dungan, K.W. and Peters, E.L.: J. Pharmac. exp. Ther. 129, 191 (1960)

5) Lish, P.M., Hillyard, I.W. and Dungan, K.W.: J. Pharmac. exp. Ther. 129, 438 (1960)

6) Drinnon, V. and Yelnosky, J.: Fedn Proc. 18, 386 (1959)

7) Manley, E.S. and Lawson, J.W.: Archs int. Pharmacodyn. Thér. 175, 239 (1968)

8) Nash, G.B., Drinnon, V. and Clark, B.B.: Fedn Proc, 17, 397 (1958)

9) Misu, Y., Tanaka, G. and Takaori, S.: Jap. J. Pharmac. 15, 176 (1965)

10) Kubo, T. and Misu, Y.: Jap. J. Pharmac. 19, 621 (1969)

11) Cox, A.R. And West, T.G.: J. Pharmac. exp. Ther. 131, 212 (1961)

12) Holland, W.C. and Ke.ern, R.L.: Circulation Res. 6, 516 (1958)

13) Shanes, A.M.: Pharmac. Rev. 10, 59, 165 (1958)

14) Tizautwein, W.: Pharmac. Rev. 15, 277 (1963) 